\title{
Remitting Seronegative Symmetrical Synovitis with Pitting Edema Syndrome Caused by Crystal-Induced Arthritis of the Wrist: A Case Report
}

\author{
Michiyuki Hakozakia,c Hironari Fukudaa,c Takahiro Tajino ${ }^{c}$ Shinichi Kikuchic \\ Satoshi Abe ${ }^{b}$ Shinichi Konno ${ }^{c}$ \\ Departments of a Orthopaedic Surgery and ${ }^{b}$ Internal Medicine, Fukushima Prefectural Minami-Aizu Hospital, and \\ 'Department of Orthopaedic Surgery, Fukushima Medical University School of Medicine, Fukushima, Japan
}

\section{Key Words}

Remitting seronegative symmetrical synovitis with pitting edema syndrome $\cdot$ Crystal-induced arthritis · Gout • Intra-articular corticosteroid injection $\cdot$ Nonsteroidal anti-inflammatory drugs

\section{Abstract}

Objective: To describe a rare case of remitting seronegative symmetrical synovitis with pitting edema (RS3PE) syndrome caused by gouty arthritis. Clinical Presentation and Intervention: A 76-year-old man presented with swelling and pain in the dorsum of feet and hands bilaterally. From the laboratory and radiologic findings, the diagnosis of gout-induced RS3PE syndrome was made. Conservative therapy with nonsteroidal anti-inflammatory drugs (NSAIDs) and intra-articular corticosteroid injection in the wrist joint completely and rapidly resolved all symptoms. The patient was successfully treated with oral administration of NSAIDs and a one-time intra-articular corticosteroid injection in the left wrist joint. Conclusion: This case demonstrated the importance of considering the possibility of crystal-induced arthritis such as gout and pseudogout, as well as malignant disease, when diagnosing the primary disease responsible for RS3PE syndrome.

Copyright $\odot 2012$ S. Karger AG, Basel

\section{Introduction}

Remitting seronegative symmetrical synovitis with pitting edema (RS3PE) syndrome is an uncommon disorder, characterized by an acute-onset seronegative inflammation with pitting edema in the distal extremities, remitting without functional impairment. RS3PE syndrome is known as a paraneoplastic syndrome caused by some solid cancers and hematologic diseases. However, RS3PE syndrome rarely occurs due to inflammatory disease including infections and crystal-induced arthritis. We describe herein an extremely rare case of gouty arthritis-induced RS3PE syndrome in a 76-year-old man.

\section{Case Report}

The patient was a 76-year-old man with no remarkable past medical history including gout/hyperuricemia. He presented with a 1-month history of swelling and mild pain in the dorsum of his right foot, and a 3-week history of the same symptoms in his left foot. In addition he had a 1-week history of swelling and moderate pain in the dorsum of both of his hands with severe pain in his left wrist. There was no history of trauma.

Upon examination, his body temperature was $38.1^{\circ} \mathrm{C}$ and local examination revealed swelling with pitting edema on the dorsum of his hands and feet bilaterally with surrounding erythema

\section{KARGER}

E-Mail karger@karger.com www.karger.com $/ \mathrm{mpp}$

\section{(c) 2012 S. Karger AG, Basel} $1011-7571 / 13 / 0223-0307 \$ 38.00 / 0$

Karger

Open access

This is an Open Access article licensed under the terms of the Creative Commons Attribution-NonCommercial-NoDerivs 3.0 License (www.karger.com/OA-license), applicable to the online version of the article only. Distribution for non-commercial purposes only.
Michiyuki Hakozaki, MD, $\mathrm{PhD}$

Department of Orthopaedic Surgery, Fukushima Prefectural Minami-Aizu Hospital 14-1 Kazashita, Nagata, Minami-Aizu-machi

Fukushima 967-0006 (Japan)

E-Mail paco@fmu.ac.jp 


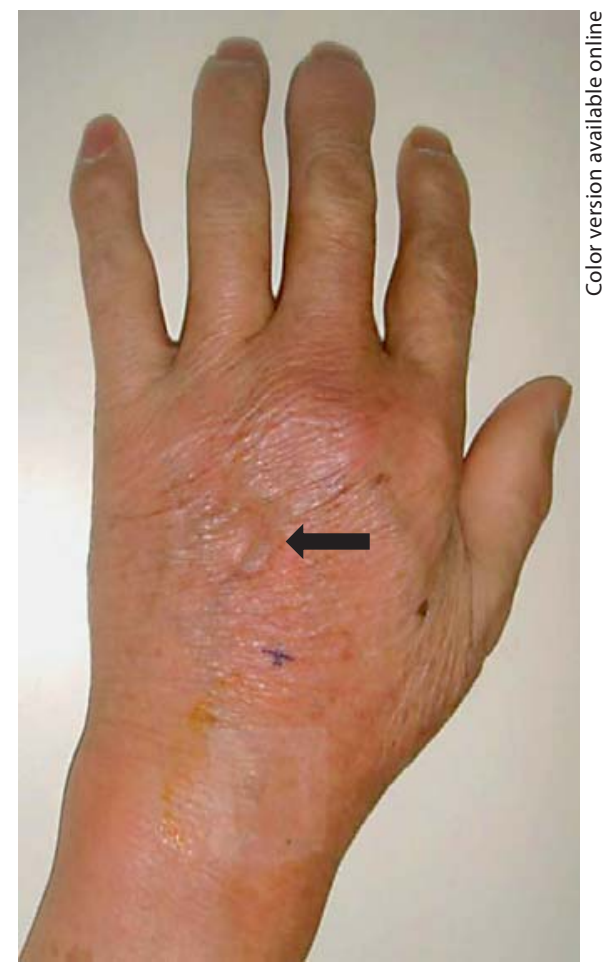

Fig. 1. Diffuse swelling with pitting edema (arrow) on the dorsum of the left hand on arrival.
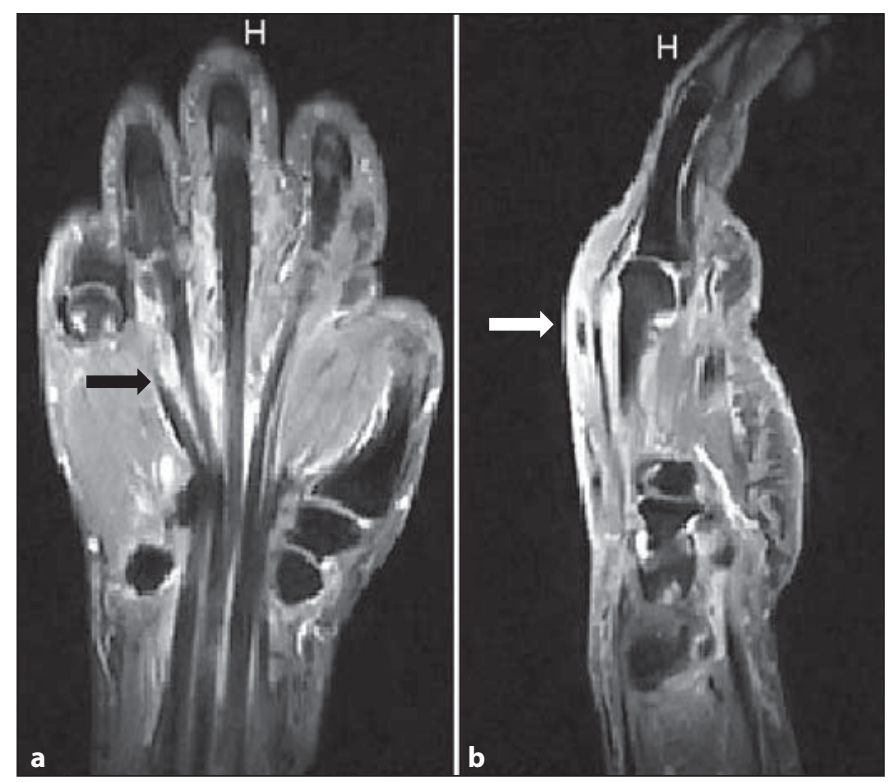

Fig. 2. Magnetic resonance imaging of the left hand reveals marked edematous, inflammatory change with diffuse enhancement around the extensor tendons (arrows) on coronal (a) and sagittal (b) gadolinium-enhanced T1-weighted fat-suppression images. and local warmth (fig. 1). His left wrist joint was severely tender, and both passive and active wrist motions were restricted because of pain. Laboratory findings revealed: white blood cell count

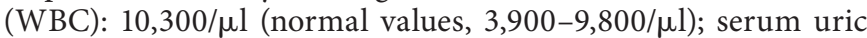
acid (UA): $5.4 \mathrm{mg} / \mathrm{dl}(3.9-8.2 \mathrm{mg} / \mathrm{dl})$; C-reactive protein: $17.1 \mathrm{mg} /$ $\mathrm{dl}(<0.3 \mathrm{mg} / \mathrm{dl})$, and erythrocyte sedimentation rate: $115 \mathrm{~mm} / \mathrm{h}$ $(<10 \mathrm{~mm} / \mathrm{h}$ in males). Several serum antibodies including rheumatoid factor, anti-cyclic citrullinated peptide antibody and other antinuclear antibodies were found to be negative. Plain radiography of his left wrist joint showed normal findings without chondrocalcinosis or osteoarthritic change. Magnetic resonance imaging of the left hand revealed findings of tenosynovitis, swelling and diffuse enhancement on gadolinium-enhanced T1weighted fat-suppression imaging, around the extensor tendons (fig. 2). The synovial fluid aspirated from his left wrist joint demonstrated both monosodium urate (MSU) and calcium pyrophosphate dehydrate (CPPD) crystals on polarized light microscopic examination. Neither gram-positive nor -negative bacteria were observed. An initial diagnosis of crystal-induced arthritis of the wrist joint and RS3PE syndrome was made. The bacterial culture results were later reported as negative. Additional laboratory and radiologic surveys were conducted to detect disease, such as malignancy, that may induce RS3PE syndrome and none revealed any abnormal findings. The patient was given a $3-\mathrm{ml}$ injection of $1 \%$ lidocaine with $0.5 \mathrm{ml}$ of $0.5 \%$ dexamethasone sodium phosphate in his left wrist joint and $180 \mathrm{mg} /$ day loxoprofen sodium hydrate orally for 1 week. Four days later, all symptoms (arthritis of the left wrist and swelling with pitting edema of both hands and both feet) had completely resolved, and his laboratory data showed decreased inflammation (WBC, $6,800 / \mu \mathrm{l}$, and $\mathrm{C}$-reactive protein, $7.90 \mathrm{mg} / \mathrm{dl})$ and increased serum UA $(7.7 \mathrm{mg} / \mathrm{dl})$. Twelve days from initial treatment, his laboratory data showed no evidence of inflammation (WBC, 5,800/ $\mu \mathrm{l}$; C-reactive protein, $<0.30 \mathrm{mg} / \mathrm{dl}$ ) and his serum UA increased to $9.2 \mathrm{mg} / \mathrm{dl}$. Moreover, a tophus was found in his left auricle (fig. 3) and resection biopsy detected MSU crystals. Finally, a diagnosis of arthritis was made on his left wrist as an acute gouty attack. He was administered allopurinol $200 \mathrm{mg}$ a day from 1 month after the remission of the joint inflammation.

The patient had no signs of recurrence during 24 months of follow-up and his serum UA level remained within normal limits.

\section{Discussion}

The present case fulfilled the original criteria of diagnosis for RS3PE advocated by McCarty et al. [1]. We ultimately made the diagnosis of RS3PE syndrome caused by crystal-induced arthritis of the left wrist, because the systemic examination for the detection of malignant disease did not show abnormality in this patient. Both MSU and CPPD crystals were detected in the synovial fluid aspirated from the left wrist. Although the coexistence of MSU and CPPD crystals in the fluid of a single joint is not uncommon [2], the correlation between the coexistence of these crystals and crystal-induced arthritis is still obscure. Though the influence of CPPD crystals (pseudo- 


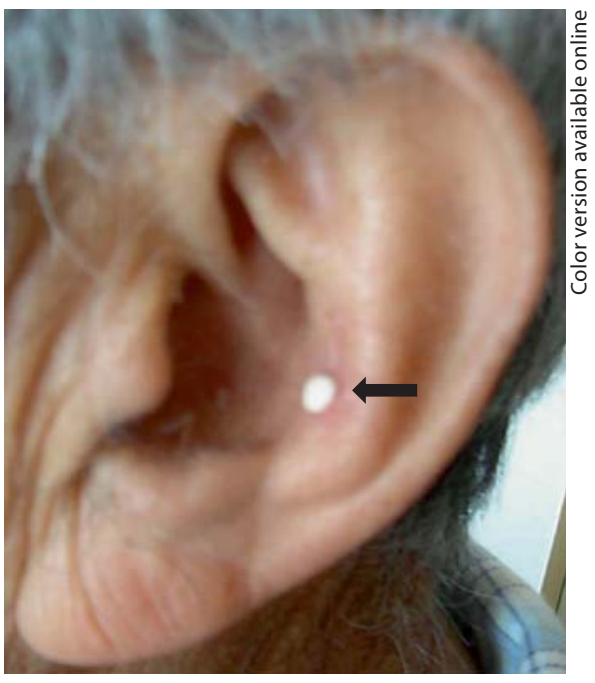

Fig. 3. Gouty tophus of the left auricle (arrow).

gouty arthritis) cannot be denied, in the present case, we speculated that this unusual presentation of symptoms was mainly caused by MSU crystals (gouty arthritis) for the following reasons: (a) plain radiography did not show chondrocalcinosis; (b) the existence of an auricular gouty tophus indicated the presence of long-standing hyperuricemia; (c) the clinical course and the serum UA levels were typical for a gouty attack, and (d) treatment with allopurinol prevented the patient from having further attacks. Currently there is no investigation that has elucidated the interaction between MSU and CPPD crystals in the same articular cavity, the mechanisms should therefore be evaluated in further clinical and basic investigations.

RS3PE syndrome caused by crystal-induced arthritis, especially by gout, is extremely rare; to the best of our knowledge, only 2 cases have been published to date [3, 4]. In the previously reported cases, the patients had formerly been diagnosed as having gout. However, they did not continue the treatment (medication) and had repeated episodes of gouty attack. As a result, gouty attack with RS3PE syndrome had occurred. In these previous cases, past medical histories of gout/hyperuricemia helped to establish the correct diagnosis of the primary disease of RS3PE syndrome. However, in the present case, the patient had not experienced a typical gouty attack and had not been diagnosed with gout/hyperuricemia. Thus, we had to diagnose circumspectly. Although RS3PE syndrome caused by gout is very rare, clinicians must consider the possibility of gout-induced RS3PE syndrome when diagnosing symmetrical inflammatory diseases because gout/hyperuricemia is a very common disorder regardless of race [5]. It may be necessary to check the past medical history and serum UA levels for a patient presenting with pitting edema of the distal extremities.

Oral administration of low-dose steroids is the current treatment of choice for patients with RS3PE syndrome, regardless of the primary disease. It is reasonable to treat the RS3PE and the primary disease simultaneously, once the primary or underlying disease diagnosis is made. Palazzi et al. [3] reported successfully treating gout-induced RS3PE syndrome with a 10-day course of meloxicam. Sugisaki and Hirose [4] reported another case of gout-induced RS3PE syndrome in which treatment with nonsteroidal anti-inflammatory drugs (NSAIDs, loxoprofen sodium hydrate and diclofenac sodium) did not show therapeutic effect and the patient was finally effectively treated with low-dose oral administration of prednisolone. In the present case, the patient was successfully treated with oral administration of NSAIDs that completely resolved all the symptoms within only 4 days, earlier than in the previous cases. To our knowledge, no report has addressed the therapeutic effectiveness of local injection of steroids for RS3PE syndrome. Since the synovium is considered to be a primary target of RS3PE syndrome [6], a one-time intra-articular injection of corticosteroids may replace oral low-dose steroid treatment. This may be particularly useful when the patient has a condition precluding oral steroid use. Furthermore, the effectiveness of the corticosteroid intra-articular injection for the present case suggests that this therapy may lead to the prompt resolution of symptoms in patients with RS3PE.

\section{Conclusion}

This case of RS3PE syndrome caused by gouty attack was successfully treated with oral NSAIDs and a onetime intra-articular corticosteroid injection in the left wrist joint. This case demonstrated the importance of considering the possibility of crystal-induced arthritis such as gout and pseudogout, as well as malignant disease, regardless of the patient's past medical history when diagnosing the primary disease responsible for RS3PE syndrome. 


\section{References}

$>1$ McCarty DJ, O’Duffy JD, Pearson L, Hunter JB: Remitting seronegative symmetrical synovitis with pitting edema. RS3PE syndrome. JAMA 1985;254:2763-2767.

-2 Chen LX, Clayburne G, Schumacher HR: Update on identification of pathogenic crystals in joint fluid. Curr Rheumatol Rep 2004; 6:217-220.
3 Palazzi C, Olivieri I, D’Amico E, Pace-Palitti V, Petricca A: Symmetrical pitting edema resembling RS3PE in gout. Clin Rheumatol 2003;22:506-507.

4 Sugisaki K, Hirose T: Remitting seronegative symmetrical synovitis with pitting edema (RS3PE) syndrome following spontaneous rupture of a gouty tophus. Mod Rheumatol 2008;18:630-633.

5 Al-Meshaweh AF, Jafar Y, Asem M, Akanji AO: Determinants of blood uric acid levels in a dyslipidemic Arab population. Med Princ Pract 2012;21:209-216.
6 Oide T, Ohara S, Oguchi K, Maruyama M, Yazawa M, Inoue K, Sekijima Y, Tokuda T, Ikeda S: Remitting seronegative symmetrical synovitis with pitting edema (RS3PE) syndrome in Nagano, Japan: clinical, radiological, and cytokine studies of 13 patients. Clin Exp Rheumatol 2004;22:91-98. 